\title{
Building a More Diverse Workforce in HIV/AIDS Research: The Time has Come
}

\author{
David M. Stoff ${ }^{1}$ - Victoria A. Cargill ${ }^{2}$
}

Published online: 2 August 2016

(c) Springer Science+Business Media New York 2016

\begin{abstract}
Investigators from diverse racial and ethnic backgrounds are grossly underrepresented in the nation's biomedical research enterprise. Projections of current demographic trends suggest that population growth rates of minority populations will outpace that of the Caucasian population by 2060 . Thus, this workforce will remain a poor reflection of the U.S. population. As a result of this underrepresentation of all sectors of the U.S. populace, the majority of the HIV research involving minority populations-those disproportionately impacted by HIV infection-will be conducted by investigators who do not resemble them. Although this does not necessarily preclude scientifically valid and important research, it produces research without the important cultural and contextual issues that can enhance the utility and generalizability of specific findings or interventions. The goal of this review is to not only raise awareness of the small numbers of minority investigators engaged in biomedical research, but also to identify the challenges to recruiting and retaining these investigators. In this article, while we discuss issues of diversity in general, the focus will be upon the mental health aspects of the HIV epidemic for illustrative purposes: to demonstrate the issues associated with enhancing
\end{abstract}

The opinions expressed in this article are those of the authors, and not necessarily those of the NIMH, NIH or the U.S. Government.

David M. Stoff

dstoff@mail.nih.gov

Victoria A. Cargill

cargillv@od.nih.gov

1 Division of AIDS Research, National Institute of Mental Health, Bethesda, USA

2 Office of AIDS Research, National Institutes of Health, Bethesda, USA investigator diversity as a strategy for remediating the chronic shortage of historically underrepresented investigators in scientific research. After presenting the magnitude of the problem and a rationale for enhancing diversity of the biomedical research workforce, we identify a number of potential reasons and challenges for the shortage of minority investigators. Aspects of the mentoring process, together with ten key suggestions, are discussed as the backdrop for the supplement papers that follow (dealing with mentoring principles, challenges, and mentoringrelated issues on mentee, mentor, mentee-mentor relationship, and programs). By identifying these realities we hope to: (1) promote greater discussions of these challenges in academic institutions and settings; (2) suggest meaningful strategies to address these challenges; and (3) foster a national discussion about the long-term investment necessary for permanent change, as there are no easy 'fixes' for these challenges.

Keywords Diversity $\cdot$ Mentoring $\cdot$ Research workforce HIV/AIDS

\section{Introduction}

Over a decade ago, the federal government announced in Healthy People 2010 its national directive to eliminate racial and ethnic health disparities by 2010 [1]. While there have been modest improvements in specific disease states, by and large, these have been outpaced by the ongoing health disparities, with significant morbidity and mortality. As the gap in life expectancy between Caucasians and African Americans and Hispanics continues to widen, a number of diseases have been identified as responsible for this trend. AIDS is one of these diseases-a bona fide 
health disparity, disproportionately impacting racial and ethnic groups, demonstrating some of the most profound disparities for African Americans, Hispanics, including other subgroups such as men of color who have sex with men [2]. The National HIV/AIDS Strategy (NHAS), released by the White House in 2011 [3], and recently updated [4], is the nation's first comprehensive and coordinated HIV/AIDS plan, identifying reduction of HIVassociated disparities.

To achieve the goal of health equity in any disease process, it is essential to understand what drives, maintains, and will remediate the disparities. A well trained and diverse scientific research workforce is an essential component of any plan to address these disparities, including disparities research (and related research areas). This diverse biomedical research workforce can increase the range and types of perspectives through which health disparities can be viewed and studied. However, to achieve such a capable diverse workforce will require a significant investment of time, effort, and resources - a daunting challenge at a time of fiscal constraints. However, this investment can be viewed as an investment in the health of America, given the significance and impact of health disparities in America [5]. An Institute of Medicine Report indicated that educational and training strategies to increase the proportion of racial and ethnic minorities in the health care workforce are a prominent approach to address the most promising health disparities interventions [5]. Achieving the goal of a highly diversified research workforce will require addressing a number of longstanding and deeply embedded challenges, as discussed by Valantine and Collins [6].

\section{The Magnitude and Nature of the Problem}

The magnitude of the problem is demonstrated in Fig. 1. The lack of diversity in the biomedical scientist population, as reflected by this data, demonstrates the potential for a narrow perspective that limits the breadth of scientific inquiry, as well as the public health responses/interventions that are derived from them. This limited diversity is particularly relevant in areas such as health disparities, where the investigators fail to reflect the populations under study, and for which the increased attention and funding have not led to a significant reduction in the scope of the problem. The lack of diversification in the scientific workforce is supported by data from several national reports [7-9]. Further, the benefits of diversification have been observed across a number of disciplinesranging from discoveries and innovations to encouraging the search for novel perspectives, leading to diversified approaches to decision making and problem solving [10, 11].

There have been a number of longstanding mentoring programs at the NIH (and other biomedical research facilities) designed to increase the number of scientists from underrepresented minorities (URM) (Table 1) with varied degrees of success, but all falling below the level anticipated. A major challenge for these programs continues to be that the increases at the training level have not been accompanied by a similar increase at the institutional level. The reasons for this are unclear but American Association of Medical College statistics from U.S. medical school faculty confirm this discrepancy [12]. Diversity in the U.S. physician workforce remains unchanged compared with prior years-with approximately $8.9 \%$ of physicians identifying as black or African-American, American Indian or Alaska Native, and Hispanic or Latino. Although blacks or African-Americans comprise $13 \%$ of the U.S. population, they account for only 4 $\%$ of the physician workforce [12].

\section{Rationale for Increasing the Number of Investigators from Underrepresented Racial and Ethnic Groups}

Despite significant scientific advancements in HIV research, these have not consistently translated into
Fig. 1 U.S. demographics and the biomedical scientist population. Sources: ${ }^{1,2}$ U.S. Census Bureau (Table 2 in Overview of Race and Hispanic Origin: 2010, and Table 6, Percent of the Projected Population by Race and Hispanic Origin for the United States: 2010 to 2050); ${ }^{3}$ Derived from data provided by NIH Office of Extramural Research Division of Information Services Reporting Branch; ${ }^{4}$ National Science Foundation, Survey of Doctorate Recipients, Appendix Table 5-17

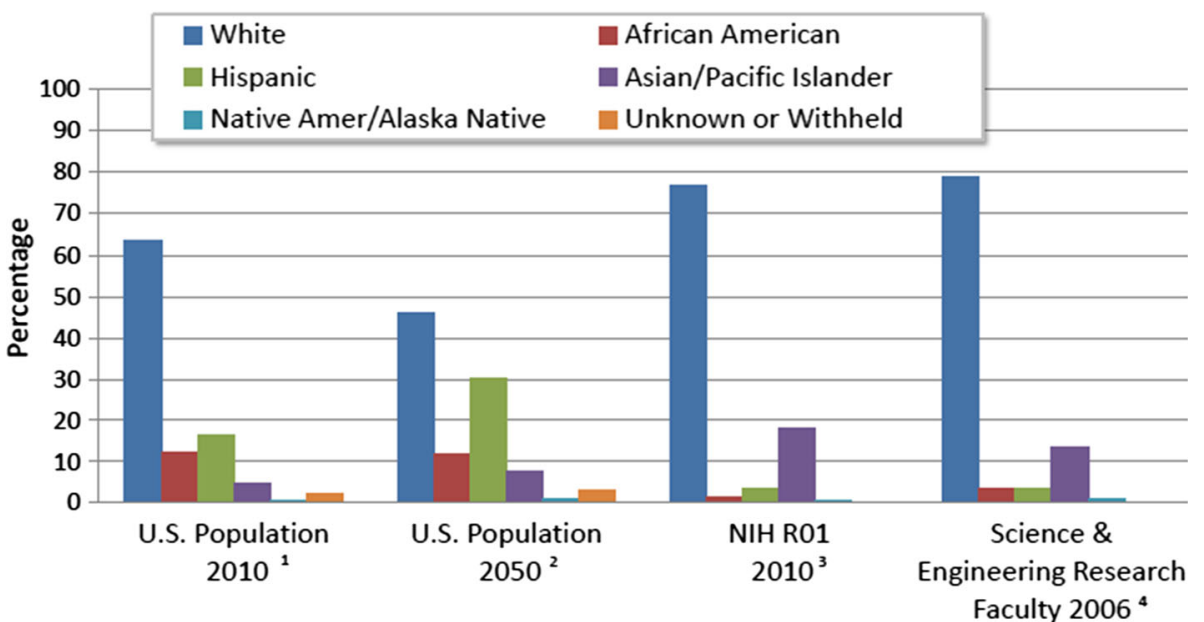


Table 1 Some barriers to success in HIV/AIDS research faced by individuals from diverse backgrounds

\begin{tabular}{|c|c|c|}
\hline Barrier & Implications of barriers & Possible solutions and strategies to overcome barriers \\
\hline \multicolumn{3}{|l|}{ Individual-level } \\
\hline Mentoring and training & $\begin{array}{l}\text { Inadequate research training opportunities, research } \\
\text { resources and pipeline programs for independent research } \\
\text { readiness; limited professional role models for career } \\
\text { planning }\end{array}$ & $\begin{array}{l}\text { Mentored research training and research education } \\
\text { awards with increased funding opportunities for } \\
\text { research training }\end{array}$ \\
\hline $\begin{array}{l}\text { Professional } \\
\text { development and } \\
\text { socialization }\end{array}$ & $\begin{array}{l}\text { Deficiencies in communication, presentation, publication, } \\
\text { grant-writing and other survival skills; deficiencies in } \\
\text { knowledge of funding sources, the grantsmanship process } \\
\text { and networking }\end{array}$ & $\begin{array}{l}\text { Mentored research training and research education } \\
\text { awards with increased funding opportunities for } \\
\text { skills building programs }\end{array}$ \\
\hline $\begin{array}{l}\text { Self-perceptions and } \\
\text { feelings }\end{array}$ & $\begin{array}{l}\text { Lack of self-confidence and isolation; unacculturated into } \\
\text { research world; relative invisibility of researchers from } \\
\text { diverse communities related to inadequate publications } \\
\text { and track record; view that diversity-focused research is } \\
\text { substandard }\end{array}$ & $\begin{array}{l}\text { Increased awareness of positive impact of research } \\
\text { contributions; broaden scope of funding agencies to } \\
\text { diversify support; encourage opportunities for } \\
\text { diverse investigators to participate in resource rich } \\
\text { programs such as Centers and/or multi-site projects }\end{array}$ \\
\hline \multicolumn{3}{|l|}{ Institutional-level } \\
\hline $\begin{array}{l}\text { Research infrastructure } \\
\text { and resources }\end{array}$ & $\begin{array}{l}\text { Lack of strong culture for research, limited availability of } \\
\text { cutting edge scientific equipment, lack of follow through } \\
\text { on institutional commitments }\end{array}$ & $\begin{array}{l}\text { Increased funding opportunities for research } \\
\text { infrastructure development; increased integration } \\
\text { of research with curriculum, improved access to } \\
\text { core facilities, increased accountability of } \\
\text { institutional leadership }\end{array}$ \\
\hline $\begin{array}{l}\text { Institutional priorities, } \\
\text { bias and support }\end{array}$ & $\begin{array}{l}\text { Overwhelming competition from large and more } \\
\text { established mainstream institutions for limited resources } \\
\text { and funding; insufficient support and/or commitment } \\
\text { from home institution }\end{array}$ & $\begin{array}{l}\text { Increased funding opportunities for collaborations } \\
\text { and partnerships; increased commitment and } \\
\text { accountability; cultivate long term relationships } \\
\text { between diverse and mainstream perspectives }\end{array}$ \\
\hline Leaky pipeline & $\begin{array}{l}\text { Unacceptable rates of attrition from one career stage to the } \\
\text { next higher one, especially at the transition points }\end{array}$ & $\begin{array}{l}\text { Increased number of programs that focus on } \\
\text { strengthening retention }\end{array}$ \\
\hline \multicolumn{3}{|c|}{ Organizational/Structural-level } \\
\hline Resources & $\begin{array}{l}\text { Inadequate time necessary to do research (administrative } \\
\text { responsibilities and teaching load); inadequate library } \\
\text { resources, such as lack of journals, to do research }\end{array}$ & $\begin{array}{l}\text { Addressing policy and political issues; protected time } \\
\text { and support; development of newer technologies }\end{array}$ \\
\hline $\begin{array}{l}\text { Competing demands and } \\
\text { inadequate incentives }\end{array}$ & $\begin{array}{l}\text { Absence of social support; health and family commitments, } \\
\text { such as childcare requirements; lack of transportation } \\
\text { options; low financial remunerations }\end{array}$ & $\begin{array}{l}\text { Addressing policy and political issues; focus on } \\
\text { contextual factors; improved organization of } \\
\text { services }\end{array}$ \\
\hline \multicolumn{3}{|l|}{ Social/cultural-levels } \\
\hline Culture and language & $\begin{array}{l}\text { Cultural insensitivity_inadequate attention to cultural } \\
\text { differences as variables of interest, available interests not } \\
\text { sensitive enough to peculiarities of diverse groups; } \\
\text { language barriers for some diverse groups in publishing } \\
\text { and completing applications }\end{array}$ & $\begin{array}{l}\text { Provide support for culturally-sensitive programs; } \\
\text { advance science of cross-cultural support; provide } \\
\text { program support for enhanced language } \\
\text { competency }\end{array}$ \\
\hline $\begin{array}{l}\text { Stigma, discrimination } \\
\text { and microaggressions }\end{array}$ & Restricted opportunities; insults and injustices & $\begin{array}{l}\text { Empowerment, partnering, rights to equality and } \\
\text { ethical imperatives }\end{array}$ \\
\hline \multicolumn{3}{|l|}{ Systemic-level } \\
\hline $\begin{array}{l}\text { Mistrust, misperceptions, } \\
\text { miscommunications } \\
\text { and insensitivity }\end{array}$ & $\begin{array}{l}\text { Stigmatization of diversity-focused research; } \\
\text { communication gaps; lack of understanding and } \\
\text { appreciation for nuances and complexities of diversity- } \\
\text { focused research }\end{array}$ & $\begin{array}{l}\text { Increased awareness and education regarding equity } \\
\text { and diversity issues; more equitable access to care } \\
\text { and research participations; increased number of } \\
\text { researchers from diverse backgrounds, especially in } \\
\text { community-based participatory research; increased } \\
\text { representation on review committees }\end{array}$ \\
\hline
\end{tabular}

advances for segments of the U.S. population disproportionately impacted by the HIV epidemic, the majority of whom are racial and ethnic minorities. This disparity in those who actually benefit from scientific advancement may place the health of minority populations at risk, as noted by the Sullivan Commission on Diversity in the
Healthcare Workforce [13]. The report concludes that the failure of the nation's health professions to keep pace with the changing demographics may be a greater cause of disparities in health access and outcomes than the persistent lack of health insurance for tens of millions of Americans. While there are no systematic studies to assess 
the contribution of the current scientific workforce representation to existing health disparities, or how new workforce models alleviating these disparities may mitigate them, it is agreed that a diverse workforce would have significant impacts on disparity-related factors and outcomes at the individual, community and systems levels [14].

Aside from addressing research to reduce and eliminate racial/ethnic disparities, there are other important reasons for increasing the diversity of the scientific biomedical workforce. These reasons have been suggested by many, and include but are not limited to: (a) broadening the research agenda by including diverse insights, perspectives and experiences to make it more relevant to the wider U.S. population; (b) ensuring full societal benefit by supporting work conducted by a more representative sample of the wider population; and (c) ensuring the full democratization of an enterprise that attempts to address the health needs of all while utilizing research conducted by very few from the populations with the poorest health indicators [5].

An example of these benefits includes lessons learned from community partnerships. We Act, a community based organization focusing on environmental justice, in partnership with Columbia University, together addressed the issue of increased asthma morbidity and mortality in Harlem. Their success demonstrates how public health research cam be strengthened and accelerated through the unique insight, experiences, and understanding that individuals from non-traditional scientific backgrounds (in this case a community) can bring [15]. For example, air quality monitors, which initially yielded few findings, were relocated in response to community input, detecting grossly elevated particulate matter. This led to the removal of the diesel powered school buses from the area, and in the process highlighted the issue of environmental disparities and dumping $[15,16]$.

To a large extent, investigators conduct research on problems that they see and feel compelled to address, which is influenced by their experiences and interests, as the lens for a researcher's curiosity is significantly influenced by their cultural and ethnic filters. First-hand involvement in the communities under study can be a benefit, as in ethnographic field research, a key prelude to prevention research [17]. The insights that individuals from underrepresented groups may possess due to their experiences, as well as the potential for greater ease in gaining acceptance and credibility in marginalized communities, may facilitate research feasibility. In addition, there is the issue of possible subjective bias in the gathering and interpretation of data that may be created by Caucasian investigators conducting research on minority populations. Advances in our knowledge of disparities can be obtained by investigators from all races and ethnicities. However, the issue of who is conducting research on disparities is related to the moral argument for diversity, which has been linked to efforts that address the inadequate representation of race, ethnicity and gender in the biomedical workforce. An Institute of Medicine symposium report stated that enhancing diversity in the health profession is not only "the right thing to do," but "the smart thing to do" [18].

\section{Challenge 1: Why is There a Significant Shortage of Research Investigators from Diverse Racial and Ethnic Backgrounds?}

There are a number of reasons why the number of research investigators from underrepresented racial and ethnic groups lags far behind Caucasians. While this supplement as well as the work of others has focused on career development/mentoring programs to overcome barriers at the individual investigator level, relatively little attention has been paid to programs that target barriers at the sociocultural and systemic levels. Although many of these barriers are more difficult to address, they nevertheless constitute significant impediments that must be considered. In Table 1, we identify some of the barriers that individuals from diverse backgrounds face at multiple levels (i.e., individual, institutional, organizational/structural, social/cultural/systemic) and suggest solutions for overcoming them. Many questions must be answered: What does the shortage of scientists from diverse backgrounds truly reflect? Is it a decreased interest in science among these populations or the financial constraints of significant post-baccalaureate debt, or the financial lure of other professions that may generate less debt and/or provide a shorter (and more assured) trajectory to success? Making science more attractive to students of all races and ethnicities, including racial and ethnic minorities through sparking a sense of excitement, has been an ongoing challenge, especially given the waning interest in this field [19].

Financial burden often discourages secondary education and post-secondary education, or leads to choosing careers that are not tied to the time and financial constraints associated with the additional training necessary for a successful research career [20,21]. The amount of postgraduate debt can be overwhelming with increasing tuition costs at both private and state supported colleges and universities. In $2011,86 \%$ of medical school graduates had a median education debt of $\$ 162,000$. A borrower with this debt level would have a repayment schedule of $\$ 1500$ to $\$ 2100$ per month after residency, depending on his or her repayment plan [22]. When the amount of outstanding premedical educational loans was analyzed, nearly twofifths of all matriculates reported they had $\$ 25,000$ or more in outstanding premedical educational loans. If one further analyzes these students by race, Blacks or African Americans $(56.0 \%)$, American Indian or Alaska Native 
$(53.7 \%)$ represented the highest percentage of those reporting the most in outstanding premedical educational loans $(\$ 25,000$ or more). The contribution of financial barriers, especially for minority populations, cannot be overstated, [23] and models that address scientific diversity pipeline issues must account for this factor. When considering strategies to remediate the barriers to research success, it will be important to include economic disincentives among the barriers that will require innovative strategies.

\section{Challenge 2: What are the Components of a Career Development/Mentoring Model to Address the Shortage of Diverse Research Investigators?}

This supplement focuses on mentoring because effective mentorship is arguably the most intense and critical form of leadership associated with training in any field. It is one of the most frequently cited components of a successful research career [24]. By extension, the absence of mentoring and research collaboration has been identified by minority investigators as critical barriers to research participation [25]. Most current models of research workforce development have targeted individual-level factors, such as mentoring, with much less attention to factors at the systems level, such as strengthening resources; promoting coordination, integration and collaboration of diversity efforts, enhancing institutional commitment and fostering leadership. Equally important is attention to the community and environmental factors that drive attrition and remediating them-including economic barriers and the social environments. Future research workforce models must target socio-economic, as well as political macrostructural factors, including the social determinants of health, such as resource disparities, discrimination, poverty, residential segregation and disparities in educational quality-many of which also overlap with those factors identified in the Healthy People 2020 [26]. Institutional contributing factors, such as institutional bias, microaggressions, policies, and procedures merit strong consideration and review, to identify whether and how these factors may contribute to the failure of individuals who may need remediation. Irrespective of the model developed, due consideration must be given to the potential effects of external factors such as: (a) the funding environment; (b) the review process; (c) the potential ramifications of a peer review process that is also confounded by a dearth of minority investigators; and (d) disproportionate burden of mentoring and other nonacademic activities on URM faculty [27-29]. Models to address the barriers and reasons for the shortage must include a number of factors, such as: (a) the impact of the total cost of debt repayment upon career choices; (b) the impact (positive and negative) of programs/ organizational culture that target investigator development across the career span and on retention in a research career; (c) the training/educational processes and how these encourage or discourage racial and ethnic students across the academic continuum; and (d) the range of pressures on URM faculty to engage in activities/roles that detract from the pursuit of a research career [30]. Several models, as discussed in this supplement, address these questions.

To address the often cited financial barriers, it would be useful to take advantage of complementary initiatives including the Gates Millennium Scholars Program [31], loan repayment programs [32], or other financial-incentive programs to improve the distribution of mentoring resources. The Gates scholarship program provides leadership development programs for minorities, and support for the cost of education by covering unmet need and selfhelp aid for minorities. The NIH loan repayment program is designed to counteract financial pressure by repaying up to $\$ 35,000$ annually of a researcher's qualified educational debt in return for a commitment to engage in NIH mission relevant research. Advances in computational methods and system sciences suggest it is possible to build models of the scientific workforce that go beyond the individual level and inform our understanding of workforce dynamics [33]. Examples of such topics that could be addressed in workforce dynamics include identifying external factors such as economic incentives and disincentives; factors that may have differential effects in various subgroups such as young investigators, minorities, women; and how short-and long-term outcomes may be rooted in personal relationships, institutions or programs.

One model worthy of note has emphasized the personenvironment fit and has been a successful career development program for Alaska Native/American Indian people [34]. Assessments of the effectiveness of this model confirm that adequate mentoring can lead to independent investigator development and funding success. The outcomes of this model would suggests that inadequate mentoring constitutes a major barrier to successful competition for NIH funding among members of underrepresented racial/ethnic groups. Data from Shavers et al. [25] also confirms that inadequate mentoring was one of the most feasible and practical actions to overcome barriers to success.

Any discussion of the diversity gap in biomedical research should also consider external factors such as the review process. An NIH working group draft report noted that "The prospect of bias in the NIH peer review process is a serious matter that calls for deliberative action in a timely fashion" [35]. The NIH report recommendations include designing an experiment that determines the effects of anonymizing applications with respect to applicant identity as well as that of an applicant's institution [35]. 
Finally, it must be noted that while mentorship is the cornerstone of career development programs, effective mentorship is multidimensional (including components such as professional networking, relevant didactic knowledge, direct research experience, scientific skills and grantsmanship. Thus any successful program must also address inadequacies in these areas [36]. The program described by Dolcini et al. [36] has been successful for minorities at the postdoctoral level, based upon conventional outcome measures such as peer-reviewed publications and funded grants in HIV prevention research. While there are many ways to define academic success, such as the creation of collaborations and networks, securing external funding and peer reviewed publications remain the most commonly used benchmarks of academic success for promotion and tenure

\section{Challenge 3: How can We Best Ensure that Racially and Ethnically Diverse Investigators Progress from One Career Stage to the Next?}

The metaphor of a "pipeline" has been used to describe the process of building a talented cadre of future scientists from diverse backgrounds. Some have questioned the pipeline metaphor by discussing the active choice aspects involved in career progression [37]. Research career development and enrichment efforts, aimed at diversifying the research workforce, are essential to foster independent research readiness. Governmental agencies, academic institutions and the private sector are working toward this goal through numerous novel programs and initiatives to build diversity. Nevertheless, there remains a significant problem as not all individuals flow equally through the pipeline; rather race, ethnicity and gender substantially influence the forward momentum through the pipeline at succeeding stages. Racial, ethnic and sexual minorities are more likely to "leak" out of the pipeline, resulting in low numbers of minorities at every stage of career development. Data from Fig. 2 demonstrate that the training pipeline functions more like a "funnel," with increased attrition among racial, ethnic and sexual minority individuals further up the academic ladder, as well as among those who proceed toward higher educational and research milestones. For example, an estimated $39 \%$ of the K-12 school age population is underrepresented racial and ethnic minorities (URM), but there is a consistent decrease in the percentage of these individuals as one progresses up the educational ladder. Only $5 \%$ of underrepresented minorities who received science and engineering doctorates (8, see Fig. 2-1). In FY 2012 only $5.3 \%$ of Principal Investigators (PIs) on NIH Research Project Grants (RPGs) were from URM groups [38]. Thus, the leaky pipeline problem appears to also be one of retention, suggesting that targeting retention at successive stages of educational and professional development could mitigate some of this attrition. The best way to determine the factors driving attrition, and at which specific pipeline stages, is to conduct careful longitudinal analyses, following the same individuals over time-and preferably from the earliest career stage possible. Targeted programs can be developed to facilitate research career development and retention by identifying those characteristics that best predict persistence and retention in biomedical research careers. Multiple etiologies have been proposed to explain the minority pipeline leak, including sociocultural and systemic factors which have received considerable attention, such as empowerment, isolation, and subtle discriminations [39].

\section{Mentoring Process to Enhance Diversity in the HIV/ AIDS Research Workforce}

When mentorship is considered for diverse researchers, another layer of complexity is added (e.g., potential impact of discrimination, stress, and isolation). One of the more salient mentorship concerns is the issue of cultural sensitivity and competency-mentees often seek mentors who can speak their language and understand their world. In view of the dominance of the Western culture in the health research world, mentors would benefit from cultural competency training to recognize the cultural values and beliefs of mentees, and may even need to go beyond cultural competency to the challenges of cultural humility and safety [40].

Another critical issue in analyzing the mentoring process (across mentee, mentor, and mentee-mentor relationship) is the use of a conceptual approach to provide a framework for mentoring strategies. For example, cognitive dynamics may be identified for the mentee through a conceptual roadmap of a research career to delineate options, stages, and trajectories to becoming an investigator [41]. A clear advantage of such an approach is treating outcomes as more than discrete dependent variables, but as a process or a pathway. One path may lead exclusively to research and others may lead to a hybrid researcher/clinician or to a program administrator or policy-maker [34]. One of the more significant conceptual illustrations of the mentoring process utilizes the notion of mentee integration, based upon the Tinto theoretical approach [42]. This conceptual model, proposed some 40 years ago, helps to explain the discouraging data showing progressively more minority attrition as the academic rank increases (Fig. 2). The central tenet is that whether an individual persists or drops out is strongly predicted by the degree of academic or social integration that evolves over time [42]. These two issues (cultural competency, conceptual basis) are examples of ways to address the mentoring process to enhance 
Fig. 2 Underrepresented minorities at different academic/career stages, 2007 Sources NCES, Digest of Education Statistics, 2008, Table 41. NSF, Women, Minorities, and persons with Disabilities, Tables A-2, C-6, E-3, and F-11. NSF, S\&E Degree Awards, 2006, Table 3. Reported in Expanding underrepresented minority participation, Washington, D.C., 2011, the National Academies Collection: Reports funded by National Institutes of Health

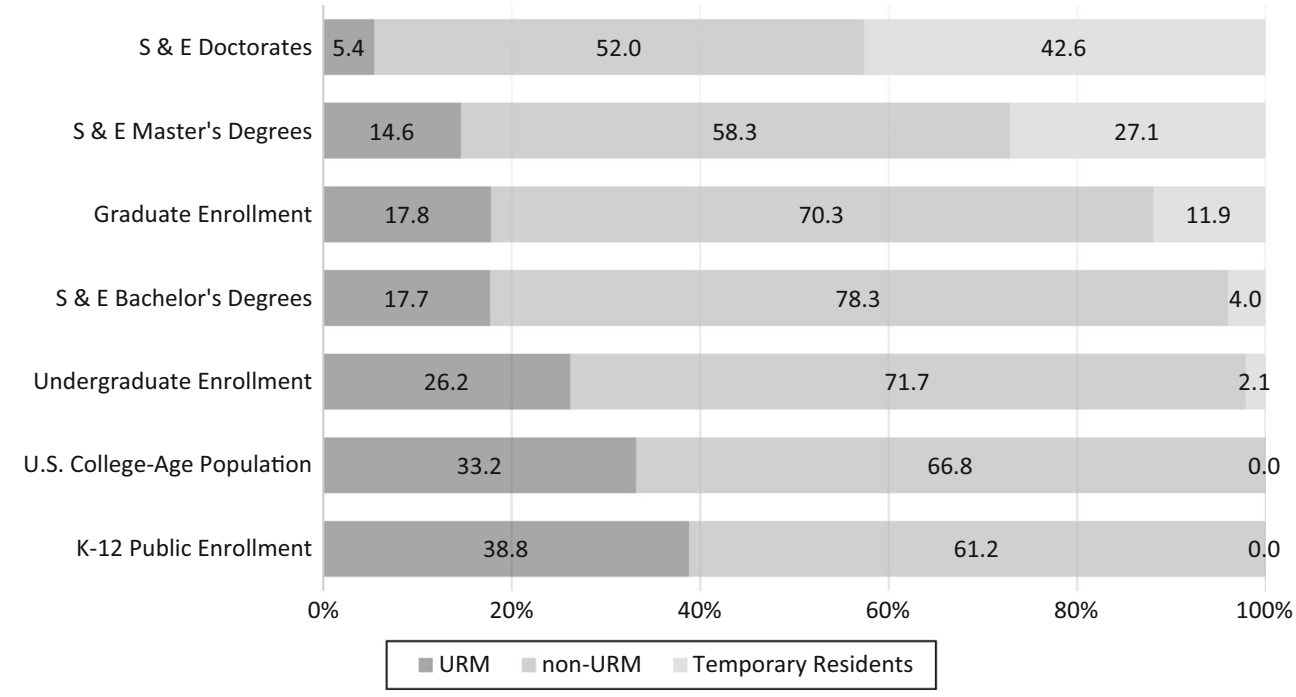

diversity; however, there are many other factors that must be taken into account. We identify ten key considerations, some of which are also embedded in the papers that follow: (1) develop evidence based, concept-driven career development programs grounded in learning theory for effective training/education and behavioral/social science research; (2) expand mentoring programs to go beyond the traditional domains of race, gender and ethnicity to include a host of other factors such as sexual orientation, language, physical disability; (3) initiate intensive recruitment, research preparation and academic enrichment programs in elementary and middle school years to continue across the high school and collegiate experience; (4) expand mentoring programs to further diversify the research workforce and within a sociocultural context, while enhancing awareness of the role of community in research; (5) leverage existing resources to develop programs with more immediate impact, utilizing existing databases for preliminary projects and senior mentors; (6) identify optimum strategies to stimulate interest in disparities-related conditions; (7) identify effective ways to recover those individuals who have dropped out of the educational system, or at risk for dropping out; (8) expand the outcome measures of successful training to non-traditional benchmarks (e.g., successful collaborations or teaching science in secondary schools); (9) develop mentoring retention programs targeted to the specific career transition points in the training pipeline to reduce attrition; and (10) provide incentives to develop and enhance an institution wide mentoring culture. Tracking programmatic impact through specific and readily quantifiable indicators of progress will be essential. This includes tracking milestones that can distinguish outcomes by career stage and by duration (short- and longer-term).

\section{This Supplement}

The focus of this supplement is to heighten awareness of the importance of enhancing, diversifying and strengthening the research workforce to reap the maximum benefits of disparities research. Papers in this supplement analyze the mentoring process from a number of perspectives for HIV investigators from diverse racial and ethnic backgrounds. Dissemination of this knowledge is an essential first step toward implementation of diversity enhancing programs.

There are three major sections for this supplement: (1) background and fundamental issues on pipeline diversification and research mentoring; (2) the mentoring process including principles, challenges and approaches; and (3) directions and future considerations for diversity mentoring programs. Although this supplement focuses on HIV/AIDS research with illustrations from this field of science, it can also be utilized to stimulate thinking for application in other health disparity states.

This paper introduces the supplement and the first section by highlighting the importance of a diverse research workforce, the challenges in trying to recruit and retain a diverse workforce, and some key considerations in the papers that follow. McGee [37] sets the stage for the other papers with theories and insights about mentoring relationships, offering a definition of mentoring, and a discussion of the importance and challenges of mentoring. Pfund et al. [43] provide a state-of-the-art review and summary of what is known about metrics for assessing mentoring.

The second section addresses some challenges and approaches of the mentoring process. Fuchs et al. [44] discuss pipeline programs for HIV undergraduate 
investigators and the theoretical frameworks that guide the mentoring approach. Brewer et al. [45] provide a perspective of the mentee from Black early-career HIV investigators, reflecting on their needs, challenges, and opportunities regarding the right mentor and mentorship environment as well as contributions and "threats" to success. Subsequent papers in the mentoring process section discuss tailored mentoring programs for Latinos and other individuals from diverse communities [46], cultural competency of the mentor in HIV research [40], multidisciplinary or transdisciplinary HIV research teams within mentoring programs [47], HIV network-based mentoring programs [48], and mentor training to improve mentee skills and outcomes [49].

The final section focuses upon next steps and directions for mentoring programs, going beyond individual level issues to considerations that may lead to career success. Manson [50] discusses how the institutional environment interfaces with mentoring programs, by focusing on organizational processes and action possibilities that are critical to promoting skills, knowledge and talent. Zea and Bowleg [51] employ an ecological framework to identify the impact of the multilevel structural barriers that impede new investigators from crossing the final frontier from mentored research to independence. Stoff and Cargill [52] close the supplement with a proposal for a general template to guide future mentoring program development.

\section{Summary}

The current HIV biomedical research workforce does not reflect the U.S. populations disproportionately impacted by the HIV epidemic. A well-trained and diverse biomedical workforce is necessary to grow a robust scientific workforce, to remain competitive in research, and to address the complex issues associated with biomedical research-all of which are needed to create a comprehensive scientific evidence base. The focus of this supplement is to heighten awareness of the importance of strengthening and maintaining the research workforce needed to realize the full benefits of disparities research. This can stimulate the dialogue necessary for the success of the mentoring programs, as well as inform stakeholders and the wider public, enhancing the impact of the mentoring program results and outcomes. Knowledge transfer and dissemination is the first step in an action plan for greater diversification of the scientific workforce through effective mentoring programs and strong outcomes. A number of potential solutions are outlined in this supplement. However, implementation will require proactive leadership, consistent support, and a commitment to enhancing/strengthening diversity pipeline programs at all training and academic levels. There is much at stake and there is no time to waste.

\section{References}

1. U.S. Department of Health and Human Services. Healthy people 2010: understanding and improving health. Washington, DC: U.S. GPO. 2nd ed. 2000. http://www.healthypeople.gov/2010/ Default.htm (updated for 2020 at http://www.healthypeople.gov/ 2010/hp2020/default.asp).

2. Wong MD, Shapiro MF, Boscardin WJ, Ettner SL. Contribution of major diseases to disparities in mortality. $\mathrm{N}$ Engl J Med. 2002;347(20):1585-92.

3. Office of National AIDS Policy. National HIV/AIDS Strategy for the United States. Washington, DC: White House; 2010.

4. Office of National AIDS Policy. National HIV/AIDS Strategy for the United States Updated to 2020. Washington, DC: White House; 2015.

5. Smedley BD, Stith AY, Nelson AR, editors. Unequal treatment: confronting racial and ethnic disparities in health care. Washington, DC: Institute of Medicine, National Academies Press; 2003.

6. Valantine HA, Collins FS. National Institutes of Health addresses the science of diversity. PNAS Early Ed. 2015;112(40):12240-2. doi:10.1073/pnas.1515612112.

7. U.S. National Science Foundation, Division of Science Resources Statistics. Women, minorities and persons with disabilities in science and engineering. Washington, DC: National Science Foundation; 2004

8. National Academy of Sciences (US), National Academy of Engineering (US), and Institute of Medicine (US) of the National Academies. Committee on Underrepresented Groups and the Expansion of the Science and Engineering Workforce Pipeline. Expanding Underrepresented Minority Participation. Washington, DC: National Academy of Sciences; 2011

9. U.S. National Science Board. The science and engineering workforce. Realizing America's potential (NSB03-69). Washington, DC: National Science Foundation; 2003

10. Page SE. The difference: how the power of diversity creates better groups, firms, schools, and societies. Princeton, Oxford: Princeton University Press; 2007.

11. Phillips KW. How Diversity Works. Sci Am. 2014;311(4):42-7.

12. American Association of Medical Colleges (AAMC). Diversity in the physician workforce: facts \& figures 2014. Section II: current status of U.S. physician workforce. http://aamcdiversity factsandfigures.org/section-ii-current-status-of-us-physicianworkforce/. Accessed 14 Dec 2015.

13. Sullivan Commission on Diversity in the Healthcare Workforce. Missing persons: minorities in the health professions. Washington, DC. 2004. www.sullivancommission.org

14. Wyatt GE, Williams JK, Henderson T, Sumner L. On the outside looking in: promoting HIV/AIDS research initiated by African American investigators. Am J Public Health. 2009;99(supplement 1):S48-52

15. Shepard PM, Northridge ME, Prakash S, Stover G. Advancing environmental justice through community based participatory research. Environ Health Perspect. 2002;110(Suppl 2):139-40.

16. Horowitz CR, Arniella A, James S, Bickell NA. Using community-based participatory research to reduce health disparities in East and Central Harlem. Mt Sinai J Med. 2004;71(6):368-74.

17. Greene MJ. On the inside looking in: methodological insights and challenges in conducting qualitative unsider research. The qualitative report, 2014;19:1-13, How To Article 15 
18. Smedley BD, Steth AY, Colburn, L and Evans $\mathrm{CH}$. The right thing to do, the smart thing to do: enhancing diversity in health professions-summary of the symposium on diversity in health professions in honor of Herbert W. Nickens, MD. Institute of Medicine. 2001

19. Hill C, Corbett C, St. Rose A. Why so few? Women in science, technology, engineering, and mathematics. American Association of University Women. Washington, DC. 2010. http://files.eric.ed. gov/fulltext/ED509653.pdf.

20. St John EP, Paulsen MB, Carter DF. Diversity, college costs, and postsecondary opportunity: an examination of the financial nexus between college choice and persistence for african americans and whites. J High Educ. 2005;76(5):545-69.

21. Malcolm LE, Dowd AC. The impact of undergraduate debt on the graduate school enrollment of STEM baccalaureates. Rev High Educ. 2012;35(2):265-305.

22. American Association of Medical Colleges (AAMC). Diversity in medical education: facts \& figures 2012. https://members.aamc. org/eweb/upload/Diversity\%20in\%20Medical\%20Education Facts\%20and\%20Figures\%202012.pdf. Accessed 3 Mar 2016.

23. Rodriguez JE, Campbell KM. Minority faculty pay a higher proportion of their earnings to student debt. Acad Med. 2014;89(3):371-2.

24. Sambunjak D, Straus SE, Marusic A. Mentoring in academic medicine: a systematic review. JAMA. 2006;296:1103-15.

25. Shavers VL, Fagan P, Lawrence D, McCaskill-Stevens W, McDonald P, Browne D, McLinden D, Christian M, Trimble E. Barriers to racial/ethnic minority application and competition for NIH research funding. J Natl Med Assoc. 2005;97:1063-77.

26. U.S. Department of Health and Human Services. Healthy People 2020. 2010. https://www.healthypeople.gov/ Accessed 18 Apr 2016

27. Espinosa L. Pipelines and pathways: women of color in undergraduate STEM majors and the college experiences that contribute to persistence. Harv Educ Rev. 2011;81(2):209-41.

28. Whittaker JA, Montgomery BJ, Martinez AVG. Retention of underrepresented minority faculty: strategic initiatives for institutional value proposition based on perspectives from a range of academic institutions. $J$ Undergrad Neurosci Educ. 2015;13(3):A136-45.

29. Sánchez JP, Peters L, Lee-Rey E, Strelnick H, Garrison G, Zhang K, Spencer D, Ortega G, Yehia B, Berlin A, Castillo-Page L. Racial and ethnic minority medical students' perceptions of and interest in careers. Acad Med. 2013;88(9):1299-307.

30. Nivet MA. Minorities in academic medicine: review of the literature. J Vasc Surg. 2010;51(4 Supplement):S53-8.

31. Gates millenium scholars. http://gmsp.org/. Accessed 15 Dec 2015.

32. NIH Loan Repayment Program. https://www.lrp.nih.gov/. Accessed 15 Dec 2015.

33. Modeling the scientific workforce (U01). http://grants.nih.gov/ grants/guide/rfa-files/RFA-GM-14-011.html. Accessed 15 Dec 2015.

34. Manson SM. Personal journeys, professional paths: persistence in navigating the crossroads of a research career. Am J Public Health. 2009;99(Supplement 1):S20-5.

35. NIH Working Group on Diversity in the Biomedical Research Workforce (WGCBRW). Draft report of the advisory committee to the director working group on diversity in the biomedical research workforce. 2012. http://acd.od.nih.gov/dbr.htm.
36. Dolcini MM, Grinstead Reznick AO, Marin BV. Investments in the future of behavioral science: the University of California, San Francisco, Visiting Professors Program. Am J Public Health. 2009;99:S43-7.

37. McGee R. Biomedical workforce diversity: the context for mentoring to develop talents and foster success within the 'pipeline'. AIDS Behav. 2016. doi:10.1007/s10461-016-1486-7.

38. NIH RePORT (Research Portfolio Online Reporting Tools), Physician Scientist Workforce (PSW) Report 2014. http://report. nih.gov/Workforce/PSW/appendix_iv_a4_2.aspx).

39. Yin HL, Gabrilove J, Jackson R, Sweeney C, Fair AM, Toto RM, for the Clinical and Translational Science Award "Mentored to Independent Investigator Working Group Committee". Sustaining the clinical and translational research workforce: training and empowering the next generation of investigators. Acad Med. 2015;90(7):861-5

40. Walters KL, Simoni JM, Evans-Campbell T, Udell W, JohnsonJennings M, Pearson CR, MacDonald MM, Duran B. Mentoring the mentors of underrepresented racial/ethnic minorities who are conducting HIV research: beyond cultural competency. AIDS Behav. 2016. doi:10.1007/s10461-016-1491-x.

41. Stead LG, Sadosty AT, Decker WW. Academic career development for emergency medicine residents: a road map. Acad Emerg Med. 2005;12:412-6.

42. Tinto V. Dropout from higher education: a theoretical synthesis of recent research. Rev Educ Res. 1975;45:89-125.

43. Pfund C, Byars-Winston A, Branchaw J, Hurtado S, Eagan K. Defining attributes and metrics of effective research mentoring relationships. AIDS Behav. 2016. doi:10.1007/s10461-016-1384-z.

44. Fuchs J, Kouyate A, Kroboth L, McFarland W. Growing the pipeline of diverse HIV investigators: the impact of mentored research experiences to engage underrepresented minority students. AIDS Behav. 2016. doi:10.1007/s10461-016-1392-z.

45. Brewer RA, Dyer T, Watson CC, Scott H. Navigating opportunities, learning and potential threats: Mentee perspectives on mentoring in HIV research. AIDS Behav. 2016. doi:10.1007/ s10461-016-1488-5.

46. Levison JH, Alegría M. Shifting the HIV training and research paradigm to address disparities in HIV outcomes. AIDS Behav. 2016. doi:10.1007/s10461-016-1489-4.

47. Magnus M, Castel A. Breaking down the siloes: developing effective multidisciplinary HIV research teams. AIDS Behav. 2016. doi:10.1007/s10461-016-1487-6.

48. Fernández MI, Wheeler DP, Alfonso SV. Embedding HIV mentoring programs in HIV research networks. AIDS Behav. 2016. doi:10.1007/s10461-016-1367-0.

49. Gandhi M, Johnson M. Creating more effective mentors: mentoring the mentor. AIDS Behav. 2016. doi:10.1007/s10461-0161364-3.

50. Manson SM. Early-stage investigators and institutional interface: importance of organization in the mentoring culture of today's universities. AIDS Behav. 2016. doi:10.1007/s10461-016-1391-0.

51. Zea MC, Bowleg L. The final frontier-transitions and sustainability: from mentored to independent research. AIDS Behav. 2016. doi:10.1007/s10461-016-1368-z.

52. Stoff DM, Cargill VA. The future HIV mentoring programs to enhance diversity. AIDS Behav. 2016. doi:10.1007/s10461-016$1502-y$. 